\title{
Survival benefit of "D2-plus" gastrectomy in gastric cancer patients with duodenal invasion
}

\author{
Koshi Kumagai $^{1} \cdot$ Takeshi Sano $^{1} \cdot$ Naoki Hiki $^{1} \cdot$ Souya Nunobe $^{1} \cdot$ Masahiro Tsujiura $^{1}$ \\ Satoshi Ida ${ }^{1} \cdot$ Manabu Ohashi ${ }^{1} \cdot$ Toshiharu Yamaguchi $^{1}$
}

Received: 9 March 2017 / Accepted: 25 May 2017 / Published online: 5 June 2017

(c) The International Gastric Cancer Association and The Japanese Gastric Cancer Association 2017

\begin{abstract}
Background The optimal extent of lymph node (LN) dissection for gastric cancer with duodenal invasion is yet to be clarified. This study sought to evaluate the significance of gastrectomy with D2-plus lymphadenectomy including posterior LNs along the common hepatic artery (no. 8p), hepatoduodenal ligament LNs along the bile duct (no. 12b) and those behind the portal vein (no. 12p), LNs on the posterior surface of the pancreatic head (no. 13), LNs along the superior mesenteric vein (no. 14v) and para-aortic LNs around the left renal vein (nos. 16a2 and 16b1) dissection. Methods Patients with gastric cancer with duodenal invasion undergoing R0 gastrectomy from January 2000 to December 2015 were enrolled. The therapeutic value index (TVI) of each LN dissection was calculated by multiplying the incidence of metastasis to each LN station by the 5-year overall survival (OS) rate of the patients with metastasis to the station.

Results In total, 117 patients were eligible. The 5-year OS rates (and TVI) of the patients with metastasis to LNs were $40.4 \%$ (7.4) in no. 12 b, $25.4 \%$ (6.8) in no. $13,32.0 \%$ (6.1) in no. $14 \mathrm{v}, 50.0 \%(13.0)$ in no. $16 \mathrm{a} 2$ and $40.0 \%(10.0)$ in no. $16 \mathrm{~b} 1$. None of the patients with metastasis in no. $8 \mathrm{p}$ or no. $12 p$ survived 5 years or longer.

Conclusion In a potentially curative gastrectomy for gastric cancer with duodenal invasion, there may be some survival benefit in dissection of nos. 12b, 13,14v, 16a2 and
\end{abstract}

Koshi Kumagai

koshi.kumagai@jfcr.or.jp

1 Department of Gastroenterological Surgery, Cancer Institute Hospital, Japanese Foundation for Cancer Research, 3-8-31 Ariake, Koto-ku, Tokyo 135-8550, Japan 16b1 LNs, while no benefit was seen in dissection of nos. $8 \mathrm{p}$ or $12 \mathrm{p}$ LNs.

Keywords Stomach - Cancer - Lymph node - Metastasis · Duodenal invasion

\section{Introduction}

Gastric cancer is the fifth most common cancer worldwide and the third most common cause of cancer death, affecting approximately 1 million new individuals each year and causing more than 700,000 deaths [1]. Survival has been prolonged by the development of chemotherapy and molecular-targeted therapy in cases of advanced gastric cancer, but surgical resection remains the most effective treatment for curable cases. Gastrectomy with D2 lymph node (LN) dissection is established as the most effective and feasible standard surgical procedure for advanced gastric cancer in Japan [2-4], and its efficacy has been proven in other countries [5-7].

Gastric cancer that located specifically in the antrum sometimes involves the duodenum. The Union for International Cancer Control (UICC) TNM classification states that the regional LNs include those of all involved sites if a tumor involves more than one site [8]. The UICC separates the duodenal LNs into pyloric, hepatic and superior mesenteric LNs [9], while the American Joint Committee on Cancer (AJCC) classifies them as hepatic, pancreaticoduodenal, infrapyloric, gastroduodenal, pyloric and pericholedochal LNs [10]. These duodenal LN regions are covered by nos. 5, 6, 12, 13 and 14 LNs in the Japanese Classification of Gastric Carcinoma [11].

The incidence of duodenal invasion in lower stomach cancer ranges from 13.5 to $33.2 \%$ [12, 13]. Tumors with 
duodenal invasion are large and deep compared to those without and have a high incidence of LN metastasis, specifically in the nodes along the proper hepatic artery and those behind the pancreas head [12, 13]. Standard surgery with curative intent for advanced gastric cancer involves resection of at least two-thirds of the stomach with a D2 LN dissection according to the Japanese treatment guidelines [4], although the optimal extent of LN dissection for gastric cancer with duodenal invasion is yet to be clarified [4]. The guidelines state that D2 plus no. 13 lymphadenectomy could be an option in curative gastrectomy for tumors invading the duodenum (Fig. 1) [4]. Number 12a LNs are included in a standard D2 gastrectomy. However, even though LNs $12 \mathrm{~b}$ and $12 \mathrm{p}$ are regarded as regional LNs of the duodenum according to the UICC/ AJCC classification, and a high incidence of "no. 12 node" involvement has been reported [12], the guidelines do not discuss the significance of dissecting no. $12 \mathrm{~b}$ or $12 \mathrm{p}$ LNs in the event of duodenal invasion. Number $14 \mathrm{v}$ LNs are not included in a D2 gastrectomy in the latest version of the treatment guidelines, but D2 plus no. $14 \mathrm{v}$ dissection has been reported to be beneficial in tumors with apparent metastasis to no. 6 nodes [4].

The objective of the current study was to evaluate the significance of D2-plus gastrectomy including dissection of the regional LNs of the duodenum, and additionally nos. $16 \mathrm{a} 2$ and $16 \mathrm{~b} 1 \mathrm{LNs}$, by assessing the incidence of metastasis to those node stations and the rates of survival.

\section{Methods}

\section{Patients}

Patients with histologically proven advanced (T2 or deeper) gastric cancer with duodenal invasion, who had undergone R0 gastrectomy at the Cancer Institute Hospital, Tokyo, Japan, from January 2000 to December 2015 were

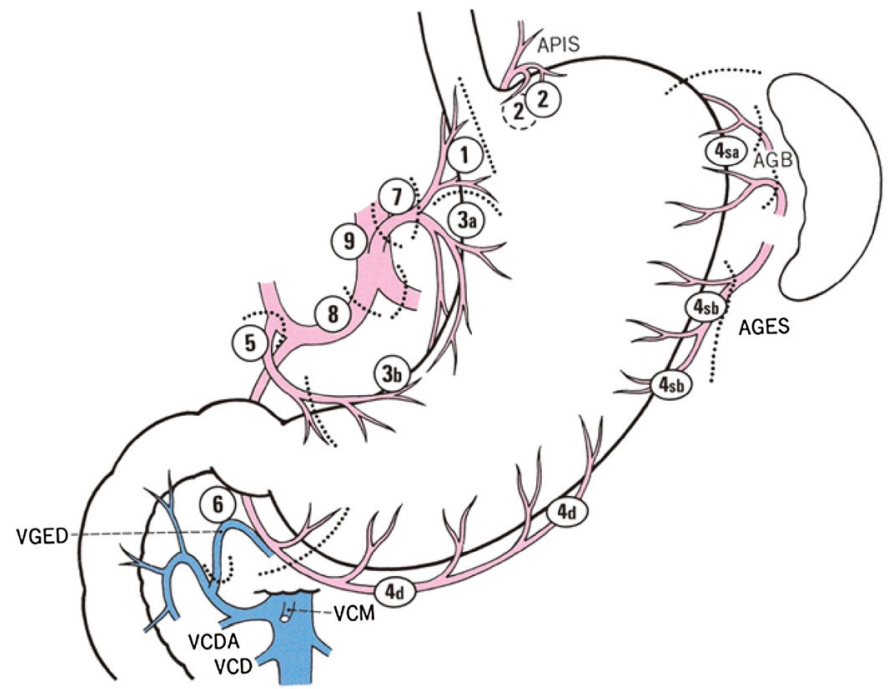

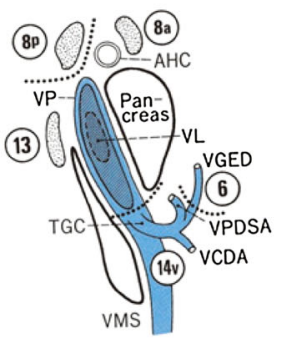

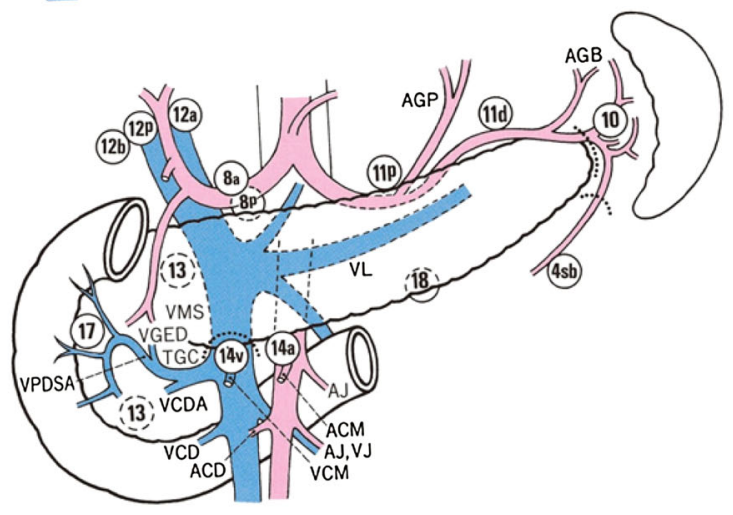

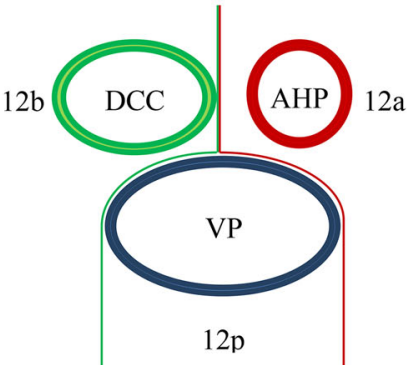

$T G C$ truncus gastrocolicus, $V C D$ vena colica dextra, $V C D A$ vena colica dextra accessoria, $V C M$ vena colica media, $V G E D$ vena gastroepiploica dextra, $V J$ vena jejunalis, $V L$ vena lienalis, $V M S$ vena mesenterica superior, $V P$ vena portae, VPDSA vena pancreaticoduodenalis superior anterior, $D C C$ ductus communis choledochus classification [11]. $A C M$ arteria colica media, $A G B$ arterias gasti breves, $A G E S$ arteria gastroepiploica sinistra, $A G P$ arteria gastrica posterior, $A H C$ arteria hepatica communis, $A H P$ arteria hepatica propria, $A J$ arteria jejunalis, APIS arteria phrenica inferior sinistra, 
enrolled in this study. Patient data were retrieved retrospectively from our prospective database and the patient's hospital records. Patients with multiple gastric cancers were excluded from the study to clarify the relationship between the primary tumor and metastasized LNs.

\section{Surgery}

In principle, D2 plus no. $13 \mathrm{LN}$ dissection has been performed at our institution for gastric cancer with duodenal invasion since its first appearance in the treatment guidelines. Because the hepatoduodenal LNs are included in the regional LNs of the duodenum, it seemed reasonable to perform en-bloc dissection of these LNs in some circumstances for both oncological and technical reasons. We therefore carried out more radical surgery in some cases, including en-bloc dissection of hepatoduodenal ligament LNs along the common bile duct (no. 12b), LNs behind the portal vein (no. 12p) and posterior LNs along the common hepatic artery (no. 8p). The no. 8p LNs are also worth noting in terms of lymphatic flow in relation to D2-plus gastrectomy for distal stomach cancer, given that they are located between the anterosuperior LNs along the common hepatic artery (no. 8a), which are frequently involved in lower stomach cancer, and the para-aortic LNs (nos. 16a2 and 16b1), which encompass lymphatic flow from the gastric cancer (Figs. 1, 2). Para-aortic lymph node dissection (PAND) was therefore additionally performed in some cases, based on the surgeon's decision. LNs along the superior mesenteric vein (no. $14 \mathrm{v}$ ) were dissected if metastasis of no. 6 was suspected. Standard D2 gastrectomy was performed in patients considered unsuitable for extensive surgery because of comorbidities.

\section{Extracted parameters}

The following parameters were extracted from the database and patient records: patient age and gender, macroscopic and histological type, surgical procedure, tumor size, depth of tumor invasion, number of retrieved LNs, number of involved LNs and survival outcome. Tumor characteristics were recorded in accordance with the Japanese Classification of Gastric Carcinoma and UICC TNM Classification $[9,11]$. The cross-sectional circumference of the stomach was divided into four equal parts: the lesser and greater curvatures and the anterior and posterior walls. Definition of LN station numbers of the stomach were recorded in accordance with the Japanese Classification of Gastric Carcinoma, 3rd edition [11] (Figs. 1, 2). In this study, stations $3 \mathrm{a}$ and $3 \mathrm{~b}$ were combined and renamed station 3 because this separation was newly introduced in the latest classification and most patients were recorded in accordance with the former classification. All the harvested LNs

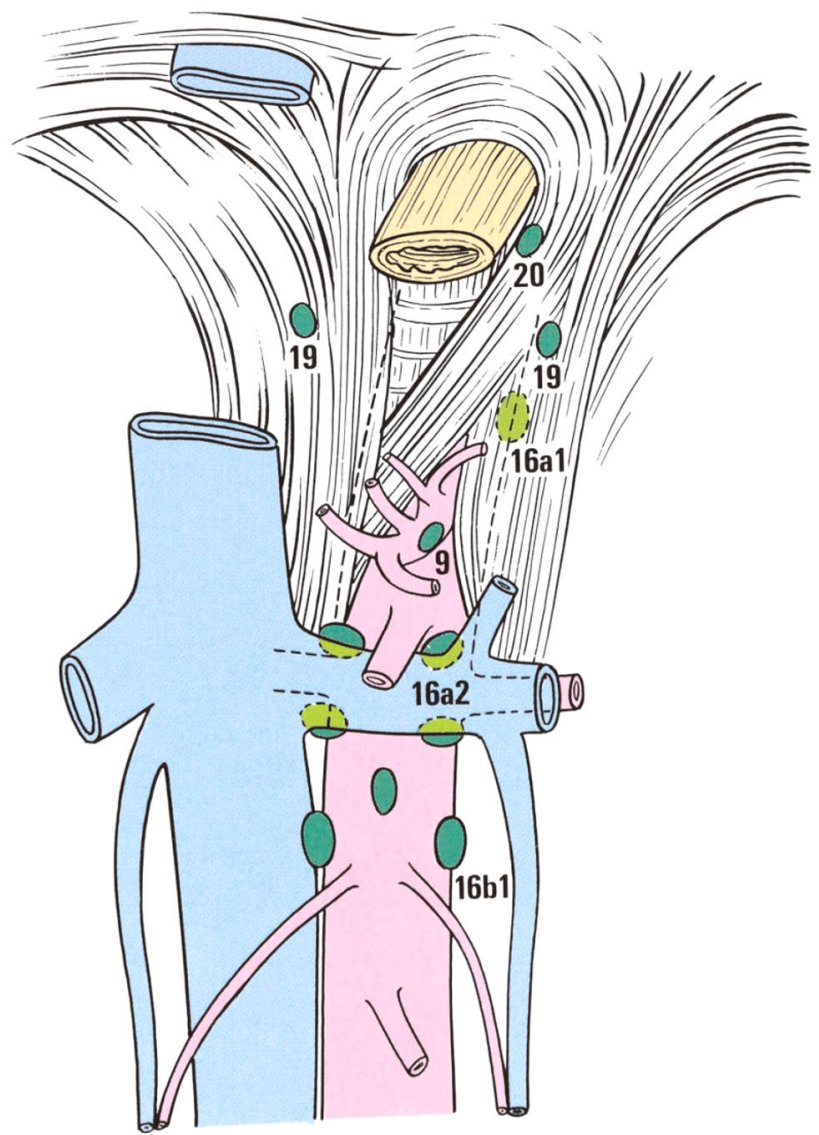

Fig. 2 Location of lymph nodes in the esophageal hiatus and in the infradiaphragmatic and para-aortic regions according to the JGCA classification [11]

were retrieved from the fresh specimen, and detection of LN metastases was conducted using hematoxylin and eosin (H\&E) staining of one section containing the largest dimension of the LN.

\section{Survival}

Overall survival (OS) was calculated from surgery to death of any cause. Kaplan-Meier curves for OS were compared between the patients with and without each $\mathrm{LN}$ station metastasis using the log-rank test.

\section{Therapeutic value of lymph node dissection}

The therapeutic value of each LN dissection was calculated by multiplying the incidence of metastasis to the station by the 5-year OS rate of the patients with metastasis to the station to give a therapeutic value index (TVI), as proposed by Sasako et al. [3]. The incidence of metastasis to each $\mathrm{LN}$ station was obtained by dividing the number of the patients who had metastases in the LN station by the number of the patients who underwent dissection of the $\mathrm{LN}$ station. 


\section{Statistical analysis}

All data are presented as the number of patients or as median (range). Statistical analyses were performed with the methods referred to above for each analysis using SPSS version 11.0 (SPSS, Chicago, IL, USA). Significance was established at $P<0.05$.

\section{Results}

During the study period, 6366 patients underwent R0 gastrectomy for gastric cancer at the Cancer Institute Hospital. Among them, 141 patients had clinically and histologically diagnosed duodenal invasion. Analyses were performed using the data from 117 patients, excluding 15 patients with pT1 gastric cancers, 8 patients with multiple gastric cancers and 1 patient with pathologically complete response to neoadjuvant chemotherapy.

\section{Clinicopathological data and perioperative findings}

Table 1 details the patient characteristics and their surgical and histological data. Neoadjuvant chemotherapy with a combination of oral fluorouracil prodrugs (S-1 or capecitabine) and cisplatin was given in six $(5.1 \%)$ patients. Adjuvant chemotherapy was given in $70(59.8 \%)$ patients, and most of the patients underwent S-1 monotherapy. The median tumor size was $61 \mathrm{~mm}$, and $85(73.3 \%)$ patients had a circumferential tumor. Serosal invasion or invasion of the adjacent organs was observed in $70(59.8 \%)$ patients. LN metastasis was observed in $93(79.5 \%)$ patients.

The extent of LN dissection varied among the 117 patients, and dissection of nos. 8p, 12b, 12p, 13 and $14 \mathrm{v}$ LNs was performed in $67,60,36,60$ and 78 patients, respectively. In terms of PALN, 23 and 20 patients underwent dissection of nos. $16 \mathrm{a} 2$ and $16 \mathrm{~b} 1 \mathrm{LNs}$, respectively.

\section{Incidence of lymph node metastasis}

Incidence of metastasis to each LN station is shown in Table 2. The incidences ranged from 0\% (nos. 4sa and 10) to $63.8 \%$ (no. 6). Regarding the LNs beyond the D2 range, nos. 12b (18.3\%), 13 (26.7\%) and 14v (19.2\%) had high incidences of metastasis.

\section{Overall survival and therapeutic value index}

Among all patients included in this study, the median follow-up was 34 months at the time of analysis. The 5 -year OS rate was $40.4 \%$ in patients with no. $12 \mathrm{~b} \mathrm{LN}$ metastasis, $25.4 \%$ in those with no. $13 \mathrm{LN}$ metastasis and
Table 1 Characteristics of patients with advanced gastric cancer and duodenal invasion undergoing curative gastrectomy

\begin{tabular}{|c|c|}
\hline Characteristic & Value \\
\hline Number of cases & 117 \\
\hline Age [median (range)] & $66(32-87)$ \\
\hline \multicolumn{2}{|l|}{ Sex } \\
\hline Male & 72 \\
\hline Female & 45 \\
\hline \multicolumn{2}{|l|}{ Neoadjuvant chemotherapy } \\
\hline Yes & 6 \\
\hline No & 111 \\
\hline \multicolumn{2}{|l|}{ Adjuvant chemotherapy } \\
\hline Yes & 70 \\
\hline No & 47 \\
\hline \multicolumn{2}{|l|}{ Surgical procedure } \\
\hline Distal gastrectomy & 108 \\
\hline Pancreaticoduodenectomy & 5 \\
\hline Total gastrectomy & 3 \\
\hline Left upper abdominal exenteration & 1 \\
\hline Tumor size [mm, median (range)] & $61(16-182)$ \\
\hline \multicolumn{2}{|l|}{ Cross-sectional circumference } \\
\hline Circumferential & 85 \\
\hline Lesser curvature & 19 \\
\hline Greater curvature & 4 \\
\hline Anterior wall & 3 \\
\hline Posterior wall & 5 \\
\hline Unknown & 1 \\
\hline \multicolumn{2}{|l|}{ Histology } \\
\hline Tubular adenocarcinoma & 42 \\
\hline Poorly differentiated adenocarcinoma & 54 \\
\hline Signet ring cell carcinoma & 15 \\
\hline Mucinous adenocarcinoma & 3 \\
\hline Others & 1 \\
\hline Unknown & 2 \\
\hline Number of retrieved lymph nodes [median (range)] & $45(12-101)$ \\
\hline \multicolumn{2}{|l|}{ Depth of invasion } \\
\hline pT2 & 28 \\
\hline pT3 & 19 \\
\hline pT4a & 61 \\
\hline pT4b & 9 \\
\hline \multicolumn{2}{|l|}{ Nodal status (JGCA Classification, 3rd English edition) } \\
\hline pNO & 24 \\
\hline pN1 (1-2) & 22 \\
\hline pN2 (3-6) & 30 \\
\hline pN3 (7-) & 41 \\
\hline
\end{tabular}

$32.0 \%$ in those with no. $14 \mathrm{v}$ LN metastasis (Table 2). The TVI values were 7.4 for no. $12 \mathrm{~b} \mathrm{LN}$ dissection, 6.8 for no. $13 \mathrm{LN}$ and 6.1 for no. $14 \mathrm{v} \mathrm{LN}$. The incidence of metastasis to no. $8 \mathrm{p}(6.0 \%)$ or no. $12 \mathrm{p}(2.8 \%)$ was low, and no one 
Table 2 Therapeutic value index (multiplication of incidence of lymph node metastasis by 5 -year survival rate) of each lymph node station in patients with advanced gastric cancer and duodenal invasion

\begin{tabular}{|c|c|c|c|}
\hline LN station number & Incidence of LN metastasis (\%) & 5 -Year OS $(\%)$ & Therapeutic value index \\
\hline 1 & $9.7(11 / 114)$ & 23.1 & 2.2 \\
\hline 2 & $11.1(1 / 9)$ & 0 & 0 \\
\hline 3 & $38.5(45 / 117)$ & 35.5 & 13.7 \\
\hline $4 \mathrm{sa}$ & $0(0 / 4)$ & - & - \\
\hline $4 \mathrm{sb}$ & $4.3(4 / 94)$ & 0 & 0 \\
\hline $4 d$ & $31.0(36 / 116)$ & 46.2 & 14.3 \\
\hline 5 & $20.5(23 / 112)$ & 24.6 & 5.0 \\
\hline 6 & $63.8(74 / 116)$ & 49.4 & 31.5 \\
\hline 7 & $21.4(25 / 117)$ & 24.6 & 5.3 \\
\hline $8 \mathrm{a}$ & $33.6(39 / 116)$ & 33.7 & 11.3 \\
\hline $8 \mathrm{p}$ & $6.0(4 / 67)$ & 0 & 0 \\
\hline 9 & $18.8(22 / 117)$ & 35.0 & 6.6 \\
\hline 10 & $0(0 / 6)$ & - & - \\
\hline $11 \mathrm{p}$ & $19.1(22 / 115)$ & 24.3 & 4.6 \\
\hline $11 d$ & $20.0(1 / 5)$ & 0 & 0 \\
\hline $12 \mathrm{a}$ & $6.9(7 / 101)$ & 47.6 & 3.3 \\
\hline $12 b$ & $18.3(11 / 60)$ & 40.4 & 7.4 \\
\hline $12 p$ & $2.8(1 / 36)$ & 0 & 0 \\
\hline 13 & $26.7(16 / 60)$ & 25.4 & 6.8 \\
\hline $14 \mathrm{v}$ & $19.2(15 / 78)$ & 32.0 & 6.1 \\
\hline $16 \mathrm{a} 2$ & $26.1(6 / 23)$ & 50.0 & 13.0 \\
\hline 16b1 & $25.0(5 / 20)$ & 40.0 & 10.0 \\
\hline
\end{tabular}

$L N$ lymph node, $O S$ overall survival with metastasis to those LNs survived to 5 years. Accordingly, the TVIs of nos. $8 \mathrm{p} \mathrm{LN}$ and $12 \mathrm{p}$ were zero (Table 2). Some patients with positive nodes in para-aortic LNs between the upper margin of the origin of the celiac artery and the lower border of the left renal vein (no. 16a2) or in those between the lower border of the left renal vein and the upper border of the origin of the inferior mesenteric artery (no. 16b1) survived for 5 years or longer, with a TVI of 13.0 for no. $16 \mathrm{a} 2$ involvement and 10.0 for no. $16 \mathrm{~b} 1$.

\section{Discussion}

This study showed the possible survival benefit and additional value of dissecting nos. 12b, 13, 14v, 16a2 and 16b1 LNs in a D2-plus gastrectomy for advanced gastric cancer with duodenal invasion. Although involvement of most of the LN stations mentioned above in gastric cancer has been regarded as a distant metastasis, results from the present study suggested they could be regarded as regional LNs in cases of gastric cancer with duodenal invasion. Indeed, it is reasonable that nos. $12 \mathrm{~b}, 13$ and $14 \mathrm{v}$ LNs are regional LNs of gastric cancer with duodenal invasion from a viewpoint of TNM classification that states that the regional LNs include those of all involved sites if a tumor involves more than one site [8].
A previous study from our institute suggested the benefit of no. $13 \mathrm{LN}$ dissection for gastric cancer patients with duodenal invasion based on the TVI evaluation [13]. The current study confirmed such a proposal with relatively high TVI in no. 13 LNs (6.8) that was equal to or better than those of level 2 LNs (nos. 7, 9 and 11p). The current study also showed a relatively high TVI (7.4) in no. 12b LNs due to better 5-year OS $(40.4 \%)$ in the patients with no. $12 \mathrm{~b}$ metastasis compared to those with no. $13 \mathrm{LN}$ metastasis $(25.4 \%)$. The high incidence of metastasis to no. 6 LNs $(63.8 \%)$ and subsequently no. 14v LNs (19.2\%), together with a relatively high 5 -year OS in patients with no. $14 \mathrm{v}$ LN metastasis $(32.0 \%)$, resulted in a relatively high TVI for no. $14 \mathrm{v}$ LNs (6.1). Notably, most patients with no. $14 \mathrm{v}$ metastasis also had no. 6 metastasis $(13 / 15,86.7 \%)$, and the 5-year overall survival among these patients was $36.9 \%$. Interestingly, the two patients with no. $14 \mathrm{v}$ metastasis without no. 6 metastasis had multiple metastases in other LN stations, including no. 13, and had very poor outcomes (4 and 13 months after surgery, respectively). Although no. $14 \mathrm{v}$ metastasis from gastric cancer is believed to occur via no. 6 metastasis, the existence of these two patients suggests that other routes of no. $14 \mathrm{v} \mathrm{LN}$ metastasis exist in patients with cancer in the duodenum. The high metastatic rate of no. 6 , strong correlation between nos. 6 and $14 \mathrm{v} \mathrm{LN}$ involvements, and the relatively high 5-year 
OS in patients with both nos. 6 and $14 \mathrm{v}$ LN metastases (36.9\%) may support the significance of no. $14 \mathrm{v}$ LN dissection in gastric cancer patients with duodenal invasion. Meanwhile, the incidence of metastasis to nos. $8 p$ or $12 p$ LNs was low, and patients with metastasis to those LN stations had extremely poor survival outcomes; no survival benefit was shown for dissection of those LNs.

It is also notable that some patients with metastasis in no. $16 \mathrm{a} 2$ or no. $16 \mathrm{~b} 1$ survived 5 years or longer, with 5 -year OS of $50.0 \%$ for no. $16 \mathrm{a} 2$ involvement and $40.0 \%$ for no. 16b1. These rates are obviously higher compared to the reported OS of 13.0 or $18.2 \%$ in patients with metastasis in those same LNs after PAND for gastric cancer $[14,15]$. Although there is evidence that D2 plus PAND does not improve survival in curable gastric cancer compared to D2 dissection alone in a randomized controlled trial (JCOG9501) [14], PAND might have some benefit in gastric cancer with duodenal invasion. In such tumors, some parts of the PALNs may be regarded as regional in duodenum-invading gastric cancer, although the retrospective nature of this study precluded the exact location of the resected PALNs being specified, and this is a limitation of this study. A prospective study with systematic D2-plus dissection is warranted to scrutinize the incidence of metastasis to each LN station and clarify the associated significance in gastric cancer with duodenal invasion.

There are some other limitations in the present study. As the indication for "D2-plus" gastrectomy had not been established and was therefore up to the surgeon, not all patients underwent dissection of the LNs beyond D2, specifically the para-aortic LNs. This might have caused uncertainties in the incidence data for metastasis and, accordingly, TVI of each LN station. TVI should be used to evaluate the therapeutic value of routinely dissected LNs (e.g., LNs included in D2 dissection) and the TVI for LNs that are not routinely dissected may be heavily influenced by selection bias. The apparently high TVI of the LNs beyond D2, specifically PALNs, might thus have been caused by selection bias, and the results for these should be interpreted with caution.

This study was also limited by the incomplete information regarding the pre- or intraoperative diagnosis of duodenal invasion. Although this is an important factor in the clinical application of D2-plus gastrectomy, the lack of this information meant that we were unable to calculate the concordance rate between the pre-/intraoperative and postoperative diagnoses of duodenal invasion.

In conclusion, this study highlighted the survival benefit and additional value in dissecting nos. 12b, 13, 14v, 16a2 and $16 \mathrm{~b} 1 \mathrm{LNs}$ in a potentially curative gastrectomy for gastric cancer with duodenal invasion, while no additional benefit was found in dissecting nos. $8 p$ or $12 p$ LNs.

\section{Compliance with ethical standards}

Conflict of interest The authors declare no conflict of interest.

Ethical statement All procedures performed in studies involving human participants were in accordance with the ethical standards of the institutional and/or national research committee and with the 1964 Helsinki Declaration and its later amendments or comparable ethical standards.

Informed consent Informed consent was obtained from all individual participants included in the study.

Human/animal rights statement This article does not pertain to any studies with animals performed by any of the authors.

Funding This study was not funded by any grant.

\section{References}

1. Ferlay J, Soerjomataram I, Dikshit R, Eser S, Mathers C, Rebelo $\mathrm{M}$, et al. Cancer incidence and mortality worldwide: sources, methods and major patterns in GLOBOCAN 2012. Int J Cancer. 2015;136:E359-86.

2. Maruyama K, Okabayashi K, Kinoshita T. Progress in gastric cancer surgery in Japan and its limits of radicality. World J Surg. 1987;11:418-25.

3. Sasako M, McCulloch P, Kinoshita T, Maruyama K. New method to evaluate the therapeutic value of lymph node dissection for gastric cancer. Br J Surg. 1995;82:346-51.

4. Japanese Gastric Cancer Association. Japanese gastric cancer treatment guidelines (ver. 4). Gastric Cancer. 2014;2017(20):1-19.

5. Sierra A, Regueira FM, Hernandez-Lizoain JL, Pardo F, Martinez-Gonzalez MA, Cienfuegos J. Role of the extended lymphadenectomy in gastric cancer surgery: experience in a single institution. Ann Surg Oncol. 2003;10:219-26.

6. Wu CW, Hsiung CA, Lo SS, Hsieh MC, Chen JH, Li AF, et al. Nodal dissection for patients with gastric cancer: a randomised controlled trial. Lancet Oncol. 2006;7:309-15.

7. Smyth EC, Verheij M, Allum W, Cunningham D, Cervantes A, Arnold D. Gastric cancer: ESMO clinical practice guidelines for diagnosis, treatment and follow-up. Ann Oncol. 2016;27:v38-49.

8. Wittekind C, Compton CC, Brierley J, Sobin LH, UICC (International Union Against Cancer). TNM supplement. A commentary on uniform use. 4th ed. Hoboken: Wiley-Blackwell; 2012.

9. Sobin LH, Gospodarowicz MK, Wittekind C. TNM classification of malignant tumours. 7th ed. Hoboken: Wiley-Blackwell; 2010.

10. Edge SB, Byrd DR, Compton CC, Fritz AG, Greene FL, Trotti A. AJCC cancer staging manual. 7th ed. Berlin: Springer; 2009.

11. Japanese Gastric Cancer Association. Japanese classification of gastric carcinoma: 3rd English edition. Gastric Cancer. 2011;14:101-12.

12. Kakeji Y, Korenaga D, Baba H, Watanabe A, Tsujitani S, Maehara Y, et al. Surgical treatment of patients with gastric carcinoma and duodenal invasion. J Surg Oncol. 1995;59:215-9.

13. Tokunaga M, Ohyama S, Hiki N, Fukunaga T, Inoue H, Yamada $\mathrm{K}$, et al. Therapeutic value of lymph node dissection in advanced gastric cancer with macroscopic duodenum invasion: is the posterior pancreatic head lymph node dissection beneficial? Ann Surg Oncol. 2009;16:1241-6.

14. Sasako M, Sano T, Yamamoto S, Kurokawa Y, Nashimoto A, Kurita A, et al. D2 lymphadenectomy alone or with para-aortic 
nodal dissection for gastric cancer. N Engl J Med. 2008;359: 453-62.

15. Tokunaga M, Ohyama S, Hiki N, Fukunaga T, Aikou S, Yamaguchi T. Can superextended lymph node dissection be justified for gastric cancer with pathologically positive para-aortic lymph nodes? Ann Surg Oncol. 2010;17:2031-6. 